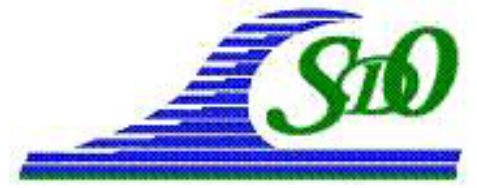

\title{
Modélisation des apports des bassins versants en baie de Bourgneuf
}

\section{Julian GILLE ${ }^{1}$, Stéphan GAILLARD ${ }^{1}$, Benoist HITIER ${ }^{2}$, Marc ROBIN ${ }^{1}$, Hélène OGER-JEANNERET ${ }^{2}$, Pascal LECOMTE ${ }^{1}$}

1. Université de Nantes, Faculté des Lettres, LETG UMR 6554 CNRS - Géolittomer, Château du Tertre, BP 81227, 44312 Nantes Cedex 3, France.

julian.gille@univ-nantes.fr

2. Ifremer, Centre de Nantes, Laboratoire Environnement Ressources Morbihan-Pays de Loire, BP 21105, 44311 Nantes Cedex 3, France.

\section{Résumé :}

Située à la jonction du domaine marin et du domaine continental, la baie de Bourgneuf est le troisième plus grand bassin conchylicole de France. La pérennisation des activités qui en découlent passe par le maintien d'une bonne qualité des eaux côtières. Ces dernières sont impactées par de multiples usages, sources potentielles de conflits et de gaspillages. Dans ce contexte, cette étude s'apparente à une démarche de type Gestion Intégrée de la Zone Côtière (GIZC) en cherchant à développer des outils pour la gestion écologique et économique durable de l'interface terre-mer. L'originalité de ce travail réside dans le couplage de trois modèles qui intègrent le continuum des milieux permettant d'évaluer (1) les flux d'eau et de matières (MES, nutriments, bactériologie, pesticide) produits en provenance du bassin versant, (2) leur transfert dans le marais qui jusqu'alors était considéré comme "une boîte noire", alimentée par des régressions linéaires et (3) leur diffusion dans la baie.

\section{Mots-clés :}

Apports continentaux - Qualité des eaux - Baie de Bourgneuf - Modélisation mathématique et couplage - GIZC

\section{Summary:}

Located at the junction of the marine field and the continental field, the bay of Bourgneuf is the third larger shellfish basin of France. The durability of the activities at the bay is linked to the maintenance from a good quality of coastal water. The coastal water are heavily impacted by multiple uses which involve conflicts and wastings. In such a context, this study leads to an Integrated Coastal Zone Management (ICZM) in seeking to develop tools for the ecological and economic management durable of the ground-sea interface. The originality of this work lies in the coupling of three models each corresponding to one of the three coastal compartments (watershed, marsh, bay). This allows to evaluate 1) flows of water and materials (MIS, nutrients, bacteriology, pesticide) coming from the watershed, 2) their transfer through the marsh which was 
before regarded as a black box and simplified with the use of linear regressions, and 3) their diffusion in the bay.

Keywords: Continental contributions - Water quality - Bourgneuf's bay - Mathematic modelisation and coupling - ICZM

\section{Introduction}

La présente étude cherche à évaluer l'impact des apports terrestres sur la qualité des eaux (MES, nutriments, bactériologie, pesticide) en baie de Bourgneuf dans le cadre du maintien des activités aquacoles et touristiques. Elle s'inscrit dans le troisième axe du projet GERRICO (http://www.gerrico.fr), piloté par l'Université de Nantes, conjointement avec l'Ifremer et financé par la région Pays de Loire. L'objectif visé est le développement d'outils d'aide pour optimiser l'exploitation des ressources marines sur le bassin conchylicole de la baie de Bourgneuf.

La mise en place d'une modélisation agro-hydrologique en zone côtière, en couplant différents modèles, constitue l'un de ces outils comme a pu le montrer notamment les études menées sur les secteurs du Croisic et de Pen-Bé (GENDRONNEAU et al., 2006) et de la lagune de Thau (PLUS et al., 2006). L'étude reprend cette démarche en y insérant la modélisation d'une zone humide littorale.

\section{Matériels et méthodes}

\subsection{Description de la zone d'étude}

La zone d'étude se situe sur la façade atlantique française, au sud de l'estuaire de la Loire (Loire-Atlantique et Vendée). Elle est constituée de trois entités géographiques distinctes : 1) le bassin versant générateur des flux, situé en amont des marais ou du littoral, 2) une zone humide littorale, lieu de transformation, de stockage et de transfert des flux avant leur rejet dans 3) la baie de Bourgneuf, réceptacle des flux continentaux et océaniques avec sa large ouverture vers le nord-ouest et l'étroit goulet de Fromentine au sud (figure 1), (VERGER, 2005).

La morphologie de cette zone est le résultat d'une succession de processus naturels et anthropiques. A partir du Mésozoïque, le socle constituant la région s'est disloqué en blocs, qui basculèrent ensuite selon un réseau de failles anciennes. La mise en place du marais et de la baie est due à l'effondrement d'un de ces blocs et à une série de transgressions marines au cours du Quaternaire (MOUNES, 1974).

Par la suite, il y a eu une baisse du niveau eustatique et la mise en place d'un cordon sableux en marge de l'actuelle zone de marais qui favorise le comblement naturel toujours actif de la baie due aux eaux turbides. Enfin, à cette succession de processus naturels s'ajoute l'action anthropique avec la poldérisation du littoral, l'aménagement du réseau de drainage pour évacuer les eaux à la mer et la construction d'ouvrages hydrauliques pour la gestion des flux d'eaux (BAUDET et al., 1987). 
Les perturbations atlantiques viennent tout au long de l'année balayer le littoral. La pluviométrie est relativement abondante et les températures sont douces. Cependant, le régime hydrologique est marqué par des étiages prononcés, conséquence de la faible surface des bassins et de la prédominance des zones de socles aux faibles réserves en eaux souterraines. Les eaux issues du bassin versant sont généralement dégradées, avec une accentuation plus marquée en période de basses eaux (juin à septembre) compte tenu des faibles étiages qui limitent la dilution des flux (ADBVBB, 2006).

Le temps de séjour des flux d'eau dans le Marais Breton peut aller de quelques heures à plusieurs mois, selon les conditions météorologiques, la période de l'année et les manœuvres hydrauliques qui en découlent. La tendance est au maintien d'un niveau d'eau bas en saison humide, pour se prémunir du risque d'inondation préjudiciable aux cultures et différer l'évacuation de l'eau, rendue complexe par le jeu des marées et la sensibilité des activités en aval. A l'inverse, en période estivale l'eau est conservée dans les fossés (BEAULIEU et al., 2005) pour assurer le maintien des fonctions écologiques du marais (biodiversité floristique et faunistique, habitats). Cet espace est reconnu comme ayant un grand intérêt en termes de biodiversité (proposé comme site d'intérêt communautaire en décembre 2003 en application de la Directive "Habitats" n92/43 du 21/05/1992 et site pilote en France pour la GIZC).

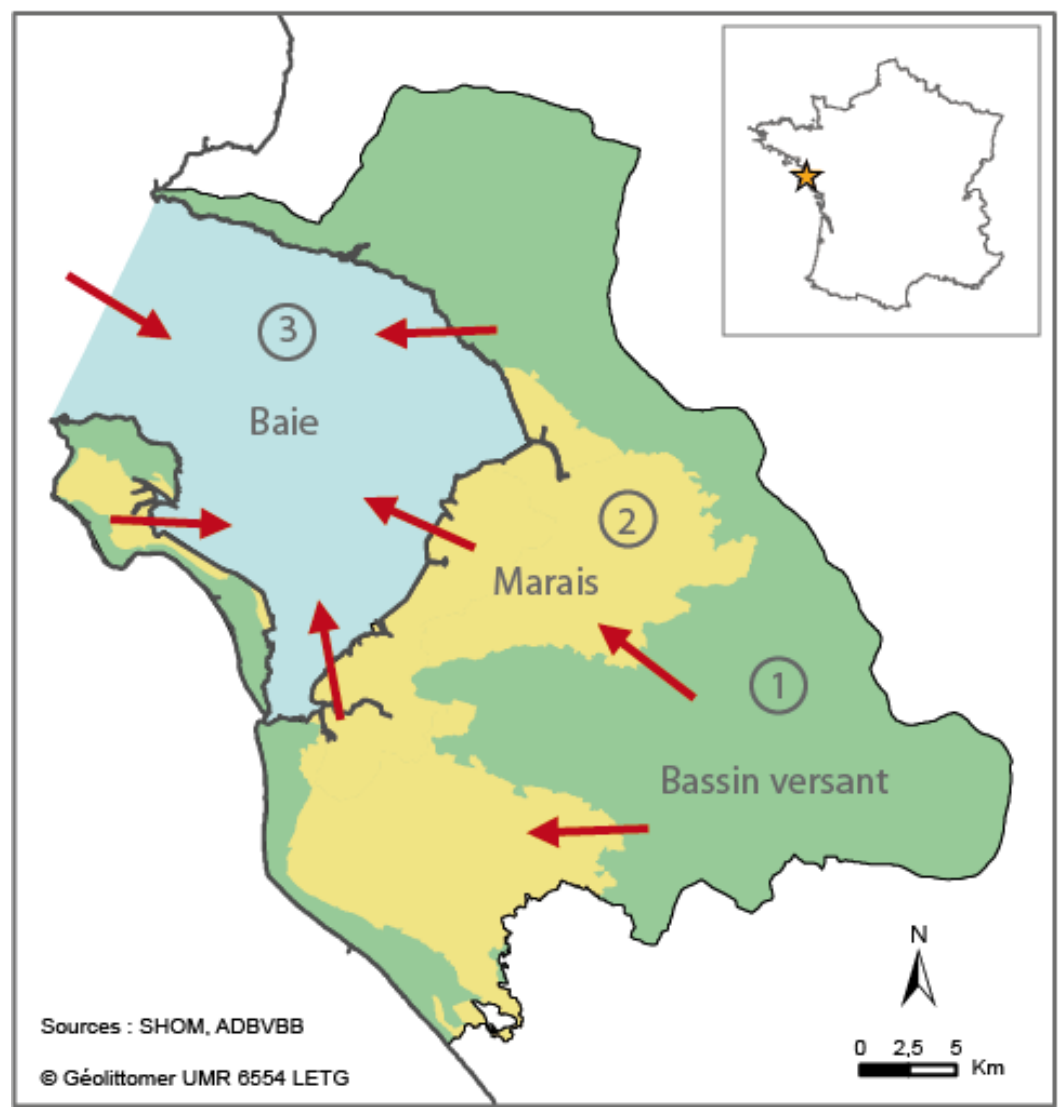

Figure 1. Direction des flux d'eau arrivant dans la baie. 


\subsection{Présentation des modèles utilisés dans chaque compartiment}

Trois modèles sont à cet effet couplés pour étudier la circulation des flux d'eau et de matières dans les compartiments qui sont traversés (figure 2).

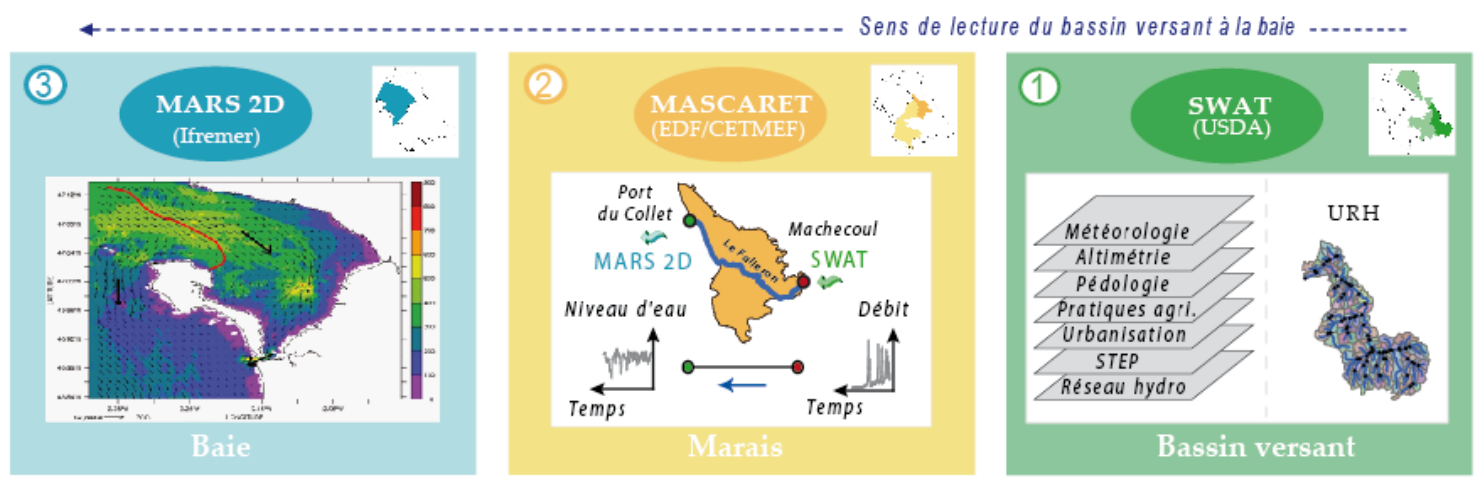

Figure 2. Chainage des modèles de circulation des flux d'eau et de matières (URH : Unité de réponse hydrologique).

Le premier modèle est le modèle agro-hydrologique SWAT® (Soil Water Assesment Tool) développé par l'Agricultural Research Service (ARS) de l'USDA (United State Department of Agriculture). Ce modèle physique semi-distribué permet de suivre, sur le bassin versant, les impacts des activités humaines sur la qualité des eaux. Les processus hydrologiques simulés dans le sol sont le ruissellement, l'infiltration, l'évapotranspiration, le prélèvement par les végétaux, l'écoulement latéral et l'écoulement vers des horizons inférieurs (NEITSCH et al., 2002). L'Unité de Réponse Hydrologique (URH) est utilisée comme unité spatiale pour les calculs. Elle constitue le résultat de la combinaison d'un type de sol, d'une classe d'occupation du sol et d'un sous bassin versant. Dans chaque URH, les volumes représentés sont le sol, l'aquifère peu profond et l'aquifère profond. Le modèle SWAT® suppose que chaque URH présente un comportement agro-hydrologique homogène et des pratiques de gestion communes (LAURENT et al., 2007). Le couplage de ce modèle avec un système d'information géographique comme ArcView ${ }^{\circledR}$ (ESRI) permet de spatialiser l'information et de visualiser les résultats.

Les sorties de ce premier modèle alimentent le modèle Mascaret ${ }^{\circledR}$ utilisé pour le marais. Il s'agit d'un modèle hydraulique monodimensionnel (1D) à surface libre basé sur les équations de Saint-Venant, développé conjointement par le Laboratoire National d'Hydraulique et d'Environnement du service R\&D d'EDF et par le Centre d'Etudes Techniques Maritimes et Fluviales (CETMEF, 2008). Il est composé de trois noyaux de calcul hydrodynamique s'appliquant aux types d'écoulement suivants : régime fluvial permanent et non permanent ou régime transcritique non permanent. Il prend en compte également des obstacles à l'écoulement tels que des écluses en renseignant leur loi de gestion. Son module Tracer offre quant à lui la possibilité de suivre l'évolution des 
phénomènes liés à la qualité de l'eau. Couplé avec le noyau hydraulique fluvial (permanent ou non) de Mascaret, il permet d'obtenir les évolutions temporelles et spatiales des concentrations en traceurs conservatifs (bactériologie ou produits phytosanitaires) ou non (nutriments) dans le réseau (DGUHC, 2007). La modélisation est axée sur les étiers de la partie nord du Marais Breton, émissaires principaux assurant la propagation des flux des bassins versants amont à la mer côtière. Chaque tronçon est renseigné par ses paramètres physiques (longueur, profils en travers,...) ainsi que par les lois hydrauliques qui les régissent en entrée et en sortie. Les conditions limites amont sont les débits et les concentrations en traceurs générés par le modèle SWAT. Les cotes d'eau enregistrées en amont de l'ouvrage à la mer sont utilisées comme conditions limites aval.

Le troisième modèle est MARS $2 \mathrm{D} \AA$, un modèle hydrodynamique développé par l'Ifremer, qui permet de suivre l'évolution des flux d'eau et de matières dans la baie. Le comportement physique du système est décrit par les équations de Saint-Venant. Il effectue le calcul des courants, des hauteurs d'eau et des concentrations en substances dissoutes dans un écoulement liquide plan, quasi-horizontal. Trois types d'informations sont nécessaires pour le faire tourner : la bathymétrie du domaine, les conditions aux limites sur les frontières ouvertes du domaine et les flux d'eau et de matières dissoutes à l'intérieur du domaine (rivières, émissaires...), (STANISIERE et al., 2006). Ce modèle mathématique utilise une technique d'emboîtement de modèles gigognes permettant de travailler à l'échelle de la baie ou du parc ostréicole (maille régulière de $75 \mathrm{~m}$ ). Les résultats sont stockés sous la forme de matrice multidimensionnelle qui permet une exploitation graphique sous forme de courbes d'évolution temporelle d'une (des) variable(s) à une maille du modèle ou de sortir des fichiers maillés à un instant donné.

\subsection{Paramètres d'entrée}

La simulation est réalisée au cours de l'automne 2006, à la suite de deux évènements pluvieux à un jour d'intervalle (le $33^{\text {ème }}$ et le $13^{\text {ème }}$ plus importants en 2006) (source : Météo France). Ces évènements pluvieux génèrent deux pics de nitrate dans les eaux à l'entrée du marais à Machecoul pendant une semaine (figure 3). L'évolution du nitrate dans le marais est modélisée pendant 30 jours le long du Falleron, en faisant les hypothèses suivantes : un seul tronçon de $17868 \mathrm{~m}$ de long, une profondeur de $3 \mathrm{~m}$ et une largeur de $8 \mathrm{~m}$ (source: Hydroconcept), une pente hydraulique de 0,03 pour mille (BAUDET et al., 1987), un coefficient de Strickler égal à 33 (BRATER et al., 1996), un réseau curé, sans pertes de charge et tous les vannages ouverts. Certains paramètres météorologiques utiles pour la modélisation des flux de nitrate sont en cours d'acquisition, et donc ils ne sont pas pris en compte à ce stade. 


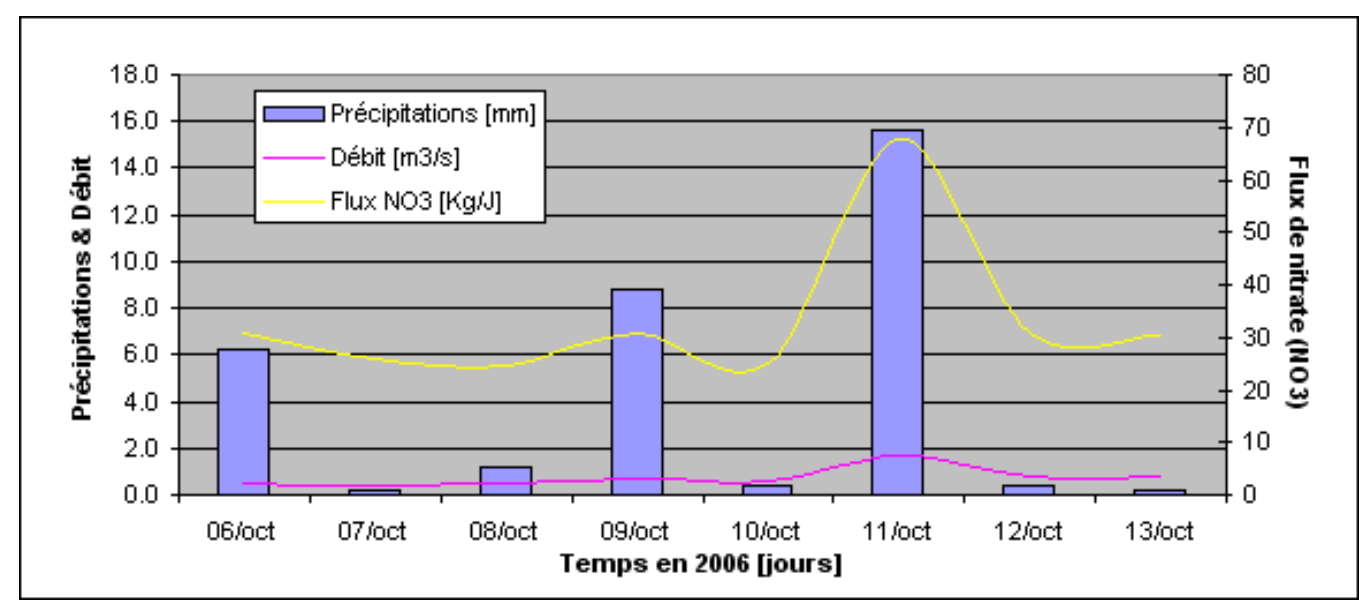

Figure 3. Précipitations et sorties du modèle SWAT.

Les résultats en sortie du marais sont ensuite injectés dans le troisième modèle avec les marées réalistes de la période allant du 01/10/2006 au 15/11/2006, un vent nul et une bathymétrie en cours de mise à jour (source : Ifremer et SHOM). Les résultats de modélisation analysés correspondent à un parc ostréicole situé à environ $3500 \mathrm{~m}$ en aval du port du Collet.

\section{Résultats préliminaires}

Les flux de nitrate sont représentés sur la figure 4 en échelle logarithmique afin de tous les visualiser. Ces résultats sont issus du couplage des trois modèles, en cours de paramétrage et de validation. Les valeurs sont donc à prendre avec précaution.

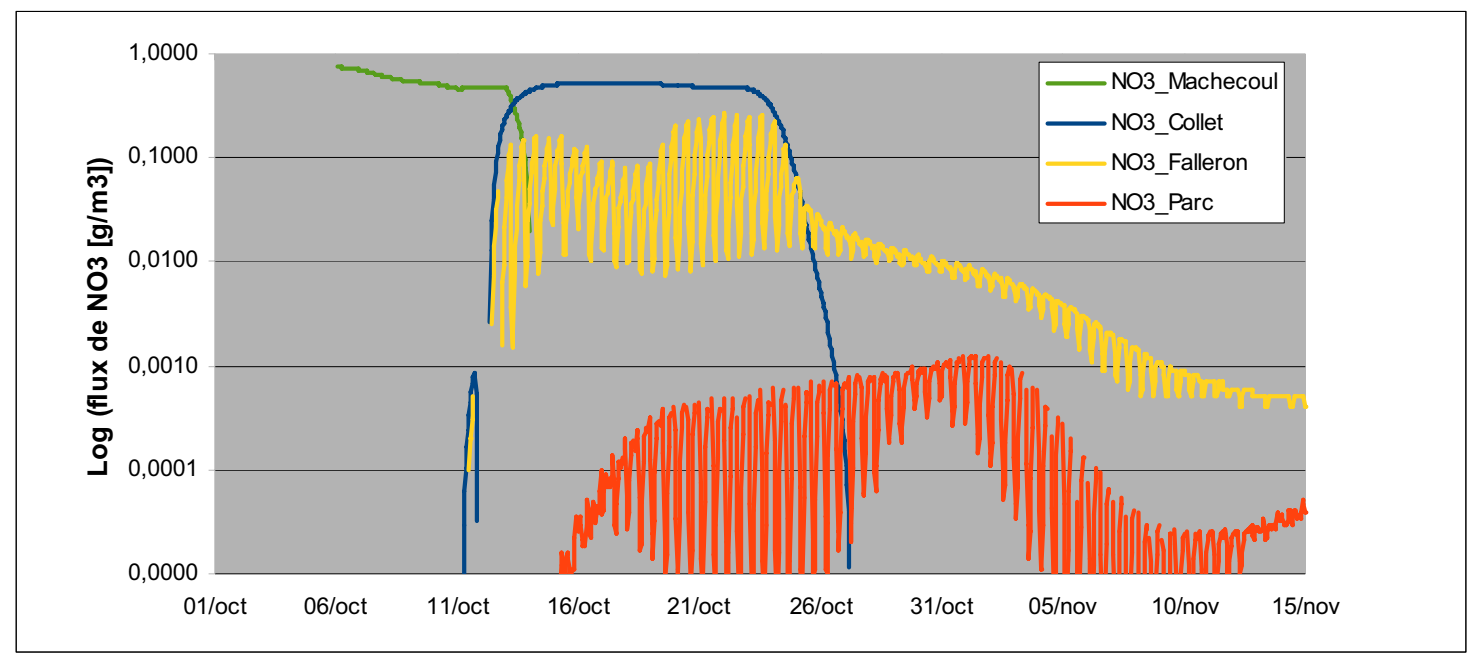

Figure 4. Evolution temporelle du nitrate dans les trois milieux.

Le nitrate arrive au port du Collet 150 heures après son apparition à Machecoul et y disparaît au bout de la $500^{\mathrm{ème}}$ heure, ce qui représente un temps de séjour de plus de 14 jours dans le marais. L'évolution des concentrations en mer suit une double sinusoïde - 
la première est semi-diurne tandis que la seconde est semi-mensuelle et se calque sur celle des coefficients de marée. Les courbes de concentrations Mascaret et Mars 2D augmentent simultanément au port du Collet mais diminuent de manière différente - la chute en rivière est "brutale" tandis que celle du coté marin est douce et sinusoïdale comme celle de la marée. Le nitrate met 8 jours à arriver sur les parcs ostréicoles à partir du port du Collet et 18 jours à partir de Machecoul. Sa dilution dans l'eau de mer est bien visible (environ 1000 fois). La concentration croît lentement pendant trois semaines environ au niveau des parcs ostréicoles mais décroît plus rapidement qu'au port du Collet. La simulation n'est pas assez longue pour voir le phénomène disparaître totalement au parc ostréicole, toutefois elle est inférieure à $10^{-4} \mathrm{~g} / \mathrm{m}^{3}$ pour une valeur proche de $1 \mathrm{~g} / \mathrm{m}^{3}$ à Machecoul. Comme spécifié précédemment, ces résultats ne peuvent pas être considérés comme réalistes. En effet, la modélisation des flux de nitrate n'indique pas un abattement dans le marais alors que les zones humides sont connues pour leur rôle auto-épurateur (FUSTEC \& LEFEUVRE, 2000). D'ici le colloque, cet aspect sera résolu.

\section{Conclusion et perspectives}

La chaîne de modèles a pour but d'évaluer l'impact des apports liés aux activités humaines sur la zone côtière et en particulier sur la conchyliculture. L'originalité de cette étude réside sur le fait 1) que la baie de Bourgneuf est une zone à forts enjeux, 2) qu'aucun travail de modélisation n'a jamais été réalisé, 3) et que le marais maritime est pris en compte dans la modélisation. D'ici juin 2010, l'objectif est de réaliser un bilan par bassin versant des flux de nutriments (nitrate, phosphate), bactériologiques (Escherichia coli), d'un phytosanitaire (glyphosate) et de suivre leur évolution dans le marais puis dans la baie de Bourgneuf. Lorsque le prototype sera calibré, des scénarii préconisateurs mis en place avec les acteurs locaux permettront d'évaluer et de tester des programmes de gestion plus respectueux de l'environnement tout en assurant la pérennité de l'activité conchylicole.

\section{Remerciements}

La mise en œuvre de ces modèles nécessite un grand nombre de données en entrée. Plusieurs établissements ou institutions ont été contactés à ce titre. Nous tenons à remercier particulièrement les Conseils Généraux de Loire-Atlantique et de Vendée, la Chambre d'Agriculture de Loire-Atlantique, les DDEA de Loire-Atlantique et de Vendée, Agro-Campus Ouest, Météo France, la CEP / SAH Sud Loire et Hydroconcept de nous en avoir donné l'accès.

\section{Références bibliographiques}

ADBVBB -Association pour le Developpement du Bassin Versant de la Baie de Bourgneuf- (2006). Etude des incidences socio-économiques de la mise en oeuvre du 
Thème 2 - Dynamique sédimentaire et transports des particules

SAGE du Marais Breton et du bassin versant de la baie de Bourgneuf. Rapport final, mars 2006, 62 p.

BAUDET J., GRUET Y., MAILLARD Y. (1987). Evolution historique des gestions hydrauliques dans le marais "breton-vendéen" - Conséquences hydrologiques et biologiques". Bull. Ecol., 18(4), pp 439-455.

BEAUliEU J.-C., CALlENS L., ANRAS L., CHASTAING C. (2005). Ouvrages hydrauliques et gestionnaires en marais atlantiques. Forum des Marais Atlantiques, Rochefort, $9 \mathrm{p}$.

BRATER E.F., KING H.W., LINDELL J.E., WEI C.Y. (1996). Handbook of hydraulics. Ed. Mc Graw Hill, 7ème édition, 640 p.

CETMEF - Centre d'Etudes Techniques Maritimes et Fluviales- (2008). Guide de prise en main de Fudaa-Mascaret 3.0. Notice ${ }^{\circ}$ GT 08.01, avril 2008, 137 p.

DGUHC -Direction Générale de l'Urbanisme de l'Habitat et de la Construction- (2007). Guide méthodologique pour le pilotage des études hydrauliques. Document principal, Ministère de l'Ecologie du Développement et de l'Aménagement Durables, septembre 2007, 58 p.

FUSTEC E., LEFEUVRE J.-C. (2000). Fonctions et valeurs des zones humides. Ed. Dunod, Technique et Ingénierie, Paris, 448 p.

GENDRONNEAU M., HAURE J., JEANNERET H., LE MERRER Y., LEQUETTE C., LOMAKINE C., MARTIN J.-L., PAPIN M., PONTHOREAU C., ROBIN M. (2006). Étude des secteurs du Croisic et de Pen-Bé : estimation des apports continentaux et évaluation des stocks conchylicoles. Fiche documentaire, Ifremer, mars 2006, 267 p.

LAURENT F., RUELLAND D., CHAPDELAINE M. (2007). Simulation de l'effet de changements de pratiques agricoles sur la qualité des eaux avec le modèle SWAT. Revue des Sciences de l'Eau, 20(4), pp 395-408.

MOUNES J. (1974). Le Marais Breton et ses Marges : Etude de Géomorphologie et de sédimentologie. Thèse de 3ème cycle, Géographie, Université de Nantes, 425 p.

NEITSCH S.L., ARNOLD J.G., KINIRY J.R., WILLIAMS J.R., KING K.W. (2002). Soil and Water Assessment Tool. Theoretical documentation, $506 \mathrm{p}$.

PLUS M., LA JEUNESSE I., BOURAOUI F., ZALDIVAR J.-M., CHAPELLE A., LAZURE P. (2006). Modelling water discharges and nitrogen inputs into a Mediterranean lagoon - Impact on the primary production. Ecological Modelling, 193(1-2), pp 69-89. doi:10.1016/j.ecolmodel.2005.07.037

STANISIERE J.-Y., DUMAS F., PLUS M., MAURER D., ROBERT S. (2006). Caractérisation des composantes hydrodynamiques d'un système côtier semi-fermé : Le Bassin de Marennes-Oléron. Ifremer - Laboratoire Environnement Ressources Poitou Charentes, décembre 2006, 112 p.

VERGER F. (2005). Marais et estuaires du littoral français. Ed. Belin, Paris, 335 p. 